\title{
ESTRESSE E VESTIBULAR COMO DESENCADEADORES DE SOMATIZAÇÕES EM ADOLESCENTES E ADULTOS JOVENS
}

\author{
Stress and the entrance ex ame as influences of somatizations \\ in young adolescents and adults
}

\section{Alice Schwanke Peruzzo ${ }^{a}$, Beatriz Cancela Cattani ${ }^{b}$, Eduardo Reuwsaat Guimarãesc, Laura de Castro Boechat ${ }^{d}$, Irani Iracema de Lima Argimone, Helena Beatriz Kochenborger Scarparof}

a Bolsista do Programa de Educação Tutorial (PET) da PUCRS, Estudante de Psicologia, Porto Alegre, RS - Brasil, e-mail: alice.peruzzo@gmail.com

b Bolsista do Programa de Educação Tutorial (PET) da PUCRS, Estudante de Psicologia, Porto Alegre, RS - Brasil, e-mail: biacattani@ portoweb.com.br

c Bolsista do Programa de Educação Tutorial (PET) da PUCRS, Estudante de Psicologia, Porto Alegre, RS - Brasil, e-mail: edurguima@yahoo.com.br

d Bolsista do Programa de Educação Tutorial (PET) da PUCRS, Estudante de Psicologia, Porto Alegre, RS - Brasil, e-mail: lauracboechat@yahoo.com.br

e Doutora em Psicologia, Professora do Programa de Pós-Graduação da PUCRS, Porto Alegre, RS - Brasil, e-mail: argimoni@pucrs.br

${ }^{\text {f }}$ D outora em Psicologia, Professora do Programa de Pós-Graduação da Faculdade de Psicologia, Tutora do Programa de Educação Tutorial (PET) e Professora da PUCRS, Porto Alegre, RS - Brasil, e-mail: scarparo@ pucrs.br

\begin{abstract}
Resumo
O presente estudo visa compreender a relação entre o Concurso Vestibular e as possíveis manifestações psicossomáticas geradas por ele. Participaram da pesquisa 141 estudantes de dois cursos pré-vestibulares particulares de Porto Alegre, durante o mês de novembro de 2006. Foram aplicados dois questionários, um contendo questões socio-demográficas e sobre a escolha profissional, e o Instrumento Inventário de Stress para Adultos de Lipp (ISSL). Na análise dos resultados, obtivemos dados sobre o nível de esgotamento psíquico e físico dos candidatos e as fases na qual eles se encontravam durante o período de preparação para o Concurso Vestibular.
\end{abstract}

Palavras-chave: Estresse; Vestibular; Psicossomática. 
Alice Schwanke Peruzzo; Beatriz Cancela Cattani; Eduardo Reuwsaat Guimarães;

\begin{abstract}
This study seeks to discover the relationship between the University Entrance examination and the possible psychosomatic manifestations which it may cause. 141 students from two private pre-entrance courses in Porto Alegre took part in the research during November 2006. Two questionnaires were used, containing socio-demographic and professional-choice questions and the Lipp Stress Symptom Inventory for Adults (ISSL). Analysis of results provided data about levels of candidates' psychic and physical exhaustion and the phases in which they were found during the Entry Examination preparation period.
\end{abstract}

Keywords: Stress; Entrance exam; Psychosomatic.

\section{INTRODUÇÃO}

\section{Adolescência e vestibular}

O exame de material teórico aponta que a adolescência tem início na puberdade, fase na qual ocorre o desenvolvimento sexual do indivíduo (Papalia \& Olds, 2000, p. 310). Nessa etapa ocorrem transformações físicas e psicológicas. É um período inusitado, no qual o jovem se depara com responsabilidades que não lhe eram atribuídas anteriormente. Tais circunstâncias podem trazer 0 enfrentamento de situações de estresse.

A conclusão do Ensino Médio e a procura por uma ocupação ou projeto profissional também estão presentes nesse momento. 0 ingresso na faculdade torna-se, assim, prioritário para muitos adolescentes. Last e Beidel (1995, p. 290) ressaltam a intensidade desse período, permeado por ansiedades, pela construção do "eu" e por perdas de atividadesinfantis em função do ingresso no mundo adulto. As expectativas referentes ao amadurecimento, independência e autonomia do indivíduo em transição são exigidas pela sociedade. D essa forma, os familiares e pessoas mais próximas ao jovem exercem influência sobre seu futuro, visto queéno ambientefamiliar eíntimo queo adolescente cria e vivencia sonhos e expectativas (Santos, 2005, p. 58). D e acordo com Hutz e Bardagi (2006, p. 65), nessa fase da vida as expectativas familiares ficam mais evidentes e os pais são vistos como modelos e fontes de valores. Por outro lado, Papalia e Olds (2000, p. 360) afirmam que durante a adolescência os jovens se afastam mais de seu núcleo familiar e dedicam seu tempo livre ao convívio com amigos, com os quais se identificam, se envolvem e pelos quais são influenciados.
Nesse momento de crescimento e transformação, o vestibular destaca-senavidadesses jovens. Trata-se de um concurso público quepermite 0 acesso ao ensino superior no Brasil, selecionando, classificando e, na maioria dos casos, eliminando quem anseia por uma vaga. A pressão sobre os estudantes é intensa, tendo em vista a dificuldade do exame, principalmente em instituições públicas, e a expectativa criada para o ingresso no ensino superior(Martins, 2004, p. 1). O sdados do Concurso Vestibular 2007 da Universidade Federal do Rio Grande do Sul são ilustrativos. Para o curso de Medicina, foram oferecidas 140 vagas para 5079 inscritos, uma média de 36 pessoas por vaga. Desconsiderando as possíveis abstenções, 97,2\% dos vestibulandos não são aprovados (Universidade Federal do Rio Grande do Sul, 2007), mostrando a inacessibilidade do curso.

Podemos afirmar que o vestibular é um ritual de passagem permeado pordesafiosacrescidos das características conturbadas da adolescência crisedeidentidade eprojeto profissional. Em função disso, é recomendado que os jovens, durante esse período, procurem uma orientação que os auxilie (Silva \& Soares, 2001, p. 117). Assim, na vida do jovem brasileiro, o exame vestibular pode ser considerado um forte gerador de estresse, que se expressa através de tensão exacerbada, diminuição de memória, irritabilidade, sonolência e perda de concentração (Rocha et al., 2006, p. 97).

\section{Psicossomática e estresse}

Nosso corpo, ao sofrer uma demanda de agentes estressores, manifesta sintomas físicos, ou seja, tende a somatizar (Campos, 1992, p. 372). 
Segundo Ballone, Neto e O tolani (2002, p. 189), a somatização aparece mais como sintoma de um sofrimento emocional do que como uma doença específica. Queixas referentes a somatizações se tornam relevantes quando culminam em alguma necessidade de tratamento médico ou interferem nas atividades do indivíduo. As manifestações somáticas mais comuns podem acometer a cabeça, as costas, as articulações, as extremidades ou causar pequenas disfunções.

Cabral et al. (1997, p. 3) postulam que doenças psicossomáticas surgem de desajustes entre os processos psicológicos e somáticos. Já Castro, Andrade e Müller (2006, p. 42), criticando a lógica cartesiana, acreditam na existência de doenças psicossomáticas que questionam a divisão entre físico e psíquico.

Entre os causadores de manifestações psicossomáticas, podemos citar 0 estresse. Popularizado no século XX , o termo foi adaptado da Engenharia, que significa "o peso que uma ponte suporta até que ela se parta" e é usado para definir sintomas causados por agentes estressores (Lipp \& Novaes, 2000, p. 12). Nessas condições, o corpo passa a secretar mais adrenalina, produzindo, assim, manifestações no organismo, formadas por componentes cognitivos, comportamentais e fisiológicos, que acabam causando uma quebra da homeostase do ser. Há diversos agentes estressores. Podem estar relacionados tanto a acontecimentos fundamentais da vida, quanto a situações cotidianas, como a perda de um objeto pessoal. As reações de estresse nocivo são proporcionais à frequência desses acontecimentos e relacionadas a fatores genéticos e ambientais. Há ainda um grupo de elementos causadores de estresse crônico, persistente e intenso. É o caso de se ter um emprego do qual não se gosta, enão poder abandoná-lo, mantendo-se por um longo tempo exposto ao agente (Margis et al., 2003, p. 66).

0 estresse tem assumido um papel relevanteno campo deestudose debates em diversos âmbitos. Essaafirmação se baseiano fato do estresse estar presente em nosso dia-a-dia. D essa maneira, é importante compreender como age no organismo e afeta a vida.

Segundo Cataldo, Gauer e Furtado (2003, p. 58), a partir do século XVIII, estudos na área da psiquiatria oportunizaram um grande aprendizado sobre o cérebro. Observou-se que esse órgão é um constante alvo de substâncias químicas que influenciam o humor e o comportamento. 0 sistema nervoso central (SNC) é fundamental no controle de substâncias secretadas através do hipotálamo, órgão que visa a homeostase corporal, controlando os processos de saciedade, além de regular os processos emocionais periféricos, ligando o sistema endócrino ao sistemanervoso. Tal ligação demonstraainfluência dos hormônios sobre a psique, como, por exemplo, a liberação de glicocorticoides e adrenalina, a fim de preparar o organismo para que receba e aja contra estímulos possivelmente ameaçadores.

Hoje não temos como preocupações maiores a busca por alimento, a caça ou a fuga de animais ferozes que possam vir a afetar nossa integridade. A maioria de nossas preocupações se dá de forma indireta, sem uma ameaça imediata à vida. 0 nível de estresse de quem recebe a notícia de que sua carreira pode estar ameaçada pode tornar-se tão grande e ser equiparável ao nível de estresse dos nossos antepassados quando se encontravam em uma situação de luta ou fuga. Segundo Cabral et al. (1997, p. 4), essa reação se define basicamente por manifestações corporais, tais como: aumento da frequência cardíaca, respiratória e da pressão arterial, entre outras.

Com o objetivo decompreender o impacto do Concurso Vestibular na adolescência, foi realizado o presente estudo. Procurou-se identificar a possível existência de vestibulandos com níveis de estresse e manifestação somática significativos.

\section{MÉTODO}

Para o delineamento, optou-se por abordagem mista transversal descritiva exploratória. A amostra foi escolhida por conveniência e constituída de 48 homens e 93 mulheres, totalizando 141 alunos, com idades entre 18 e 24 anos e matriculados em um dos dois cursos pré-vestibulares escolhidos em Porto Alegre.

Os instrumentos de pesquisa foram: um questionário sócio-demográfico e 0 Inventário de Sintomas de Stress para Adultos de Lipp (ISSL). Esse instrumento de pesquisa identifica sintomas de estresse, classificando-o como físico ou psicológico e categorizando o sintoma prevalente (Calais, S. L., Andrade, L. M. B. \& Lipp, M. E. N, p. 260). A intenção dessa aplicação foi identificar o estresse, a fase em quese encontrava esuasformas de expressão.

A análise do questionário deu-se de forma quantitativa e qualitativa. Qualitativamente, se analisou, através da Análise de Conteúdo (Bardin, 
1991), as expectativas dos pais quanto à aprovação dos filhos, atividades de lazer, sentimentos referentes ao período de estudos e sintomas físicos apresentados. A etapa quantitativa abrangeu informações sobre o sexo do participante, idade, ano em que concluiu o ensino médio, escola em que estudou, curso escolhido, concursos para os quais já prestou vestibular, preferência por universidade, se cursaria uma universidade particular no caso de uma aprovação, tempo diário de estudo, se tinha atividades de lazer e com qual frequência.

Para facilitar a análise, 11 grupos foram criados para reunir os cursos oferecidos pelo Ensino Superiorno Brasil citados pelos estudantes. 0 critério utilizado foi a semelhança na área de atuação e a demanda. Assim, Medicina, Odontologia e Direito formam um grupo cada. $\mathrm{O}$ restante foi reunido da seguinte forma: Engenharias (da computação, civil, ambiental, mecânica, de produção, metalúrgica, elétrica, de alimentos, de materiais, de minas, química e de controle e automação), área da Saúde (E ducação Física, Farmácia, Nutrição, Enfermagem e Fisioterapia), das Ciências Humanas (Administração, História, Ciências Sociais, Psicologia e Letras), das Ciências Exatas (Economia, Arquitetura, Contabilidade, Física, Ciência da Computação e Ciências Aeronáuticas), das Ciências Biológicas (Biologia, Biomedicina e Veterinária), das Ciências da Terra (G eologia e G eografia) e das Comunicações e Artes (Publicidade e Propaganda, Relações Internacionais, Relações Públicas, D esign, Jornalismo, Teatro e Artes Plásticas). Ainda foi criada a categoria "Mais de um curso" para aqueles que citaram de primeira opção dois ou mais cursos, não especificando preferência por nenhum.

Ressalta-se que as informações foram coletadas no mês de novembro de 2006, devido à proximidade com o início do vestibular e período de maior estresse dos estudantes.

A análise dos dados quantitativos foi realizada através da Estatística D escritiva (média, mediana, moda, desvio padrão, máximo, mínimo, frequências e percentuais) e o Teste do Qui Quadrado, com o nível de significância de 5\% (p d" 0,05). Os dados foram computados no Programa SPSS, versão 11.5 for W indows.

Na coleta dos dados, os vestibulandos não foram questionados se sofriam de alguma patologia que pudesse influenciar seus níveis de estresse ou se estavam em tratamento psicológico ou farmacológico, o que é um viés a se considerar no presente estudo.

\section{RESULTADOS E DISCUSSÃO}

Nas Tabelas 1 e 2, geradas pelo programa SPSS, apresentamos alguns dados quantitativos da pesquisa. Elas contêm informações sobre os candidatos que prestaram vestibular entre o fim do ano de 2006 e o início do ano de 2007, como o curso que optaram no Ensino Superior e a quantidade de vezes que prestaram vestibular.

O grupo pesquisado é de uma população jovem, com média de 19,05 anos de idade. Tal dado está relacionado com o critério deidade estabelecido para a coleta dos dados: idade mínima 18 anos e máxima 24 anos.

Comparando o tipo de escola que os participantes estudaram, nota-se que apenas 20\% (28 alunos), aproximadamente, tiveram algum contato com instituições públicas ao longo da sua formação escolar. Esse dado nos revela que os cursinhos utilizados para o levantamento de dados possuem um perfil de alunos com significativo poder aquisitivo que, além de pagarem o curso prévestibular particular, tiveram a possibilidade de umaformação escolartambém em estabelecimentos de ensino particular.

TABELA 1 - Curso de escolha dos pré-vestibulandos

\begin{tabular}{lcc}
\hline $\begin{array}{l}\text { Para qualcurso } \\
\text { vai prestar } \\
\text { vestibular }\end{array}$ & Frequência(n) & Percentual (\%) \\
\hline Medicina & 39 & \\
Odontologia & 11 & 27,7 \\
Direito & 10 & 7,8 \\
Engenharia & 14 & 9,1 \\
Outras áreas da saúde & 6 & 4,3 \\
Outras áreas humanas & 15 & 10,6 \\
Outras áreas exatas & 12 & 8,5 \\
Ciências biológicas & 10 & 7,1 \\
Ciências da terra & 3 & 2,1 \\
Comunicação eartes & 16 & 11,3 \\
Mais de um curso & 5 & 3,5 \\
\hline Total & $\mathbf{1 4 1}$ & $\mathbf{1 0 0 , 0}$ \\
\hline
\end{tabular}

Pode-se observar que mais de um quarto da amostra deseja cursar Medicina. Tal dado pode evidenciar que, além da tradição histórica, essa profissão está relacionada com prestígio, possibilidade de alta remuneração e, principalmente, status. 
Ainda em relação à tabela acima, percebe-se uma grande variedade de opções de cursos no Ensino Superior do Brasil. Esse fato tende a gerar uma maior possibilidade para estudantes experimentarem diferentes cursos e de realizarem a prova do vestibular para diferentes universidades. Fora analisado, também, a quantidade de vezes que os participantes haviam realizado a prova do vestibular para um curso diferente do que pretendiam cursar na época da aplicação do apêndice.

TABELA 2 - Número de vezes que prestou prova de vestibular

\begin{tabular}{ccc}
\hline $\begin{array}{l}\text { Quantas vezesjá } \\
\text { realizou prova } \\
\text { devestibular }\end{array}$ & Frequência (n) & Percentual (\%) \\
\hline 0 & 37 & 26,2 \\
1 & 27 & 19,1 \\
2 & 33 & 23,4 \\
3 & 18 & 12,8 \\
4 & 13 & 9,2 \\
5 & 3 & 2,1 \\
6 & 6 & 4,3 \\
7 & 2 & 1,4 \\
9 & 1 & 0,7 \\
25 & 1 & 0,7 \\
\hline Total & $\mathbf{1 4 1}$ & $\mathbf{1 0 0}$ \\
\hline
\end{tabular}

Os dados gerados pela Tabela 2 evidenciam que a maior parte dos estudantes que participaram do estudo nunca haviam prestado vestibular. Tal dado está relacionado, também, ao fato de que a maioria dos estudantes tinha 18 anos na data da aplicação do questionário, idade na qual normalmente os jovens começam a realizar as provas.

As Tabelas 3 e 4 apresentam respostas coletadas através do Inventário de Sintomas de Stress paraAdultos de Lipp eindicam se há presença de estresse entre os vestibulandos, que fase de estresse é, e qual seu tipo. A proximadamente 61,7\% dos participantes apresentaram alguma forma de estresse, enquanto apenas $38,3 \%$ não 0 manifestaram de forma alguma.
TABELA 3 - Classificação do tipo de estresse apresentado pelos participantes

\begin{tabular}{lcc}
\hline Tipode estresse & Frequência(n) & Percentual (\%) \\
\hline Psicológico & 58 & 41,1 \\
Físico & 16 & 11,3 \\
Ambos & 13 & 9,2 \\
Nenhum & 54 & 38,3 \\
\hline \multicolumn{1}{c}{ Total } & $\mathbf{1 4 1}$ & $\mathbf{1 0 0}$ \\
\hline
\end{tabular}

A forma predominante do estresse apresentado foi a psicológica. Mesmo assim, um número bastante expressivo dealunosnão apresentou forma alguma de estresse ao longo do período de preparação para as provas, mostrando, dessa forma, que o estresse não é sempre uma manifestação recorrente nos estudantes pré-vestibulandos.

TABELA 4 - Fase de estresse

\begin{tabular}{lcc}
\hline Tipo de estresse & Frequência (n) & Percentual (\%) \\
\hline Alerta & 1 & 0,7 \\
Resistência & 82 & 58,2 \\
Q uase exaustão & 3 & 2,1 \\
Exaustão & 1 & 0,7 \\
Nenhum & 54 & 38,3 \\
\hline \multicolumn{1}{c}{ Total } & $\mathbf{1 4 1}$ & $\mathbf{1 0 0}$ \\
\hline
\end{tabular}

Observou-se que 54 participantes $(38,3 \%)$ não apresentaram estresse e 87 participantes $(61,7 \%)$ tinham estresse. Desse segundo grupo, um participante encontrava-se na Fase de Alerta, 82 participantes (94,2\%) encontravam-se na Fase de Resistência, três na Fase de Quase Exaustão e um na Fase de Exaustão. Ainda sobre o grupo que apresentou estresse, quanto à prevalência dos sintomas, 16 participantes $(18,3 \%)$ tiveram prevalência de sintomas físicos, 58 participantes $(66,6 \%)$ prevalência de sintomas psicológicos e 13 participantes (15\%) tiveram igual prevalência em ambos.

Verificou-se, dessa forma, uma alta taxa de pré-vestibulandos estressados. Esse dado comprova que 0 vestibular tende a gerar casos de estresse. Entretanto, a prevalência de sintomas psicológicos 
Alice Schwanke Peruzzo; Beatriz Cancela Cattani; Eduardo Reuwsaat Guimarães; 324 Laura de Castro Boechat; Irani Iracema de Lima Argimon; Helena Beatriz Kochenborger Scarparo

aponta que nem sempre a manifestação do estresse é física, podendo ser psicológica, como no caso dos vestibulandos que participaram da pesquisa.

D os 141 pré-vestibulandos que participaram da pesquisa, a grande maioria $(60 \%)$ dissenão termanifestado sintomasfísicosno decorrer do mês de novembro de 2006, demonstrando, bem como os dados do Inventário apontam, que a somatização não é algo fundamentalmente presente entre os estudantes. D entre aqueles que expressaram sofrer de mal-estar físico (55 jovens), 69,1\% sofriam de males de caráter interno (aqueles relacionados com órgãos internos do corpo), como dores de cabeça e úlcera. Sintomas externos (associados com mudanças visíveis do corpo), como ganhar peso ou problemas referentes à pele, foram citados por $9,1 \%$ dos estudantes.

Através da análise dos resultados, observou-se a associação entre o estresse e o sexo $(p=0,00)$. Concluiu-se que as mulheres sofreram mais de estresse do que os homens. Isso pode basear-se no fato de que, com a abertura cada vez maior do mercado detrabalho para o sexo feminino, a sociedade exige das mulheres uma grande carga de atividades, onde a carreira é acrescida de exigências pessoais, biológicas, sexuais e sociais. Esses dados são alarmantes e de grande importância, não só para as mulheres em si, mas também para as famílias e a sociedade, considerando o grande papel da mulher e das mães para a sociedade (Calais, Andrade \& Lipp, 2003, p. 262).

As mulheres estressadas representam 49,6\% dos participantes que responderam aos questionários. O s mesmos autores ainda apontam 0 concurso vestibular como um possível fator gerador de estresse, já que a maioria dos adolescentes e adultos jovens se vê diante da escolha profissional nesse momento. A forma como o indivíduo lida com essa situação estressante pode ser um fator importante para o sucesso ou não no concurso.

Rocha et al. (2006, p. 98), em seu estudo relacionado com adolescentes em momento de escolha profissional, encontraram maior incidência de sintomas depressivos e estresse em estudantes do sexo feminino. Além de apontar os fatores sociais e culturais como possíveis causas desse resultado, os autores afirmam que o fato de a mulher ter sido privada do mundo acadêmico até pouco tempo pode ter sido um fator influenciador. A evidente transformação corporal, as mudanças hormonais, os sentimentos de vergonha, medo, a descoberta da sexualidade e os tabus impostos, tanto pela família quanto pelo meio em que vivem, são alguns dos aspectos que tornam o período da adolescência um momento conflituoso para as mulheres.

A análise dos dados ainda permitiu identificar uma associação entre o estresse e 0 tipo de universidade de preferência do vestibulando, podendo esta ser pública ou particular $(p=0,056)$. Dentre o grupo de participantes que expressaram predileção por alguma universidade (125 estudantes), a maioria desses demonstrava preferir as Federais (90,8\%). Essa evidência pode estar relacionada com o fato de que nesse tipo de universidade há uma maior liberdade financeira, já que 0 acesso ao estudo acadêmico é gratuito. Levenfus (1997, p. 201), em seu estudo com vestibulandos, aponta que o nível socioeconômico do indivíduo exerce grande influência na escolha da universidade.

Além disso, 58\% dos estudantes que apresentavam predileção por algum tipo de universidade sofriam de algum tipo de estresse, que pode estar associado com a grande concorrência que as universidades federais apresentam para se ingressar em um de seus cursos. D ados adicionais também podem corroborar essa prevalência. A tradição de estudar numa universidade federal e 0 status queisso proporciona, por muitas vezes oferecer um ensino mais qualificado, ainda são pensamentos comuns e exercem influência no ambiente social.

Nesse âmbito, vale a pena ressaltar que os participantes da pesquisa estudavam em cursos pré-vestibulares privados, ou seja, pagavam para receber a educação e a preparação adequada para o concurso vestibular. Fica evidente que a busca pela aprovação em universidades públicas também éum objetivo da classemédia e médiaalta. Andrade (2002, p. 7) acredita que o modelo de educação superior está imensamente relacionado com a formação escolardo indivíduo. No Brasil, as escolas públicas vêm enfrentando um enorme problema quanto à qualidade do ensino oferecido. As escolas privadas (particulares) têm proporcionado uma educação mais esmerada e prepara seus alunos para a entrada no curso superior. Não há, portanto, uma igualdade de oportunidade, uma vez que 0 sistema de ensino público no Brasil encontra-se defasado, pois tal se mostra deficiente em relação à entrada de seus alunos no ensino superior.

Outro dado importante encontrado foi a relação entre estresse e horas de estudo $(p=0,048)$. A maioria dos estudantes que sofria de estresse estudava 
de duas a três horas por dia, além das horas que estudavam no curso pré-vestibular $(17,5 \%$ dos questionários). Além disso, notou-se que os alunos que estudam menos horas por dia apresentam menos estresse do que os que estudam mais tempo. Esse resultado pode estar relacionado com o fato de que os alunos que se dedicam menos ao vestibular, não se sintam pressionados a serem aprovados e, por isso, fiquem mais tranquilos e relaxados antes do período das provas. Os fatores emocionais têm uma grande importância dentro da sintomatologia proveniente da somatização. De acordo com Ballone (2002, p. 191) 0 que pode ser feito para atenuar tais sintomas é expressar verbalmente as emoções, preocupações, angústias e sentimentos para que esses sintomas não se manifestem fisicamente, isto é, é preciso uma conscientização dos pacientes sobre a importância de seu estado emocional sobre seu estado físico. 0 nosso corpo segue o princípio da homeostase, buscando 0 equilíbrio interno através da autorregulação.

Além disso, observou-se uma dependência entre 0 estresse e 0 aparecimento de sintomas físicos $(\mathrm{p}=0,00)$. Tal situação ficou evidenciada pelo fato de que 32,1\% daqueles participantes que foram classificados como estressados haviam apresentado algum sintoma físico durante o mês de novembro de 2006, tais como dores de cabeça, dores no corpo, problemas digestivos, entre outros.

Durante a análise dos dados coletados, constatou-se a existência numerosa de estudantes que não se inseriam no grupo dos considerados estressados. Estes eram estudantes que apresentavam manifestações de sintomas físicos, sem indício de estresse ou, ao contrário, com a presença de estresse, mas ausentes de somatização.

D e acordo com Lipp e Novaes (2000, p. 12), o médico austríaco Hans Selye definiu a existência de três fases de estresse (por ele também chamado de "Síndrome de Adaptação G eral"): a Fase de Alarme, a de Resistência e a de Exaustão, seguidas em ordem, respectivamente. D evemos citar, contudo, que a autora introduziu mais uma fase no Inventário de Sintomas de Stress para Adultos, chamada de Q uase Exaustão. A Fase de Alerta ocorre quando o indivíduo entra em contato com 0 agente estressor e manifesta algumas sensações típicas como taquicardia e sudorese excessiva, as quais são um aviso de que há um certo desequilíbrio interno. Estafase é importante para que o corpo reaja e enfrente a situação, garantindo sua sobrevivência.
Através da associação entre o estresse e a fase na qual ele se encontra ( $p=0,00)$, obteve-se uma maior prevalência dos vestibulandos estressados na Fase de Resistência (94,2\%), a segunda fase, na qual, após o desequilíbrio ocorrido inicialmente na Fase de Alerta, tenta-se atingir novamente a harmonia interna através da adaptação ou da eliminação dos agentes estressores. D evido ao grande gasto de energia, épossível haver desgastes e cansaço excessivos, esquecimentos e autodúvidas. Se, no entanto, o indivíduo não conseguir reequilibrar suas forças, este pode passar para a fase seguinte, a de Exaustão (ou de Quase Exaustão, de acordo com o Inventário) e ser passível de um maior comprometimento físico e psíquico que pode resultar em doenças (Lipp, 2000, p. 13).

Fora observado também a dependência entre o estresse e a prevalência de seus sintomas ( $p$ $=0,00)$. Dentre os 87 estudantes que estavam acometidos de estresse, 41,1\% sofriam de estresse psicológico, 11,3\% de estresse físico e 9,2\% de ambos. O estresse psicológico, de acordo com Lipp (2000, p. 18), produz apatia, depressão, falta de ânimo, ansiedade e sentimentos referentes à raiva. Evidencia-se, assim, que o estresse causado pelo vestibular não tende a causar necessariamente somatizações nos jovens. Ele acaba causando muito mais desconforto emocional do que físico.

A análise dos questionários demonstrou haver associação entre o sexo do participante e as fase do estresse $(p=0,00)$. D entre os participantes considerados estressados (87), 94,2\% deles estavam na fase de resistência. As mulheres representavam 79,2\% dos membros do grupo de resistência. Conclui-se que o sexo feminino é mais acometido de estresse do que o masculino, pois esteve prevalente em todas as fases de estresse.

No que diz respeito à análise qualitativa, alguns pontos merecem ser destacados. Ao serem questionados so bre as expectativas que os familiares eamigos manifestavam em relação a seu desempenho no vestibular, 77,3\% dos estudantes relataram sentir expectativas positivas de seus pares. Algumas expressões como "todos acreditam quevou passar", "acreditam no meu potencial" e "apostam em minha capacidade" foram citadas pelos participantes.

Em contrapartida, quando questionados sobre os sentimentos que o vestibular provoca, $83 \%$ dos jovens manifestaram sensações negativas como "ansiedade", "medo", "insegurança", 
Alice Schwanke Peruzzo; Beatriz Cancela Cattani; Eduardo Reuwsaat Guimarães; 326 Laura de Castro Boechat; Irani Iracema de Lima Argimon; Helena Beatriz Kochenborger Scarparo

"aflição". Apenas 4,3\% apresentaram impressões positivas, como "confiança", "responsabilidade" e "animação".

Em relação às atividades de lazer, os jovens demonstraram dedicar mais tempo a atividades de entretenimento, como ir ao cinema, sair com amigos eir afestas. Estasforam apontadas por $31,2 \%$ dos jovens. Já $22 \%$ dos estudantes relataram que têm os esportes como atividade em seu tempo livre, praticando academia, atividades em grupo e caminhadas.

\section{CONSIDERAÇÕES FINAIS}

Através deste trabalho, foi possível melhor compreender de que forma os estudantes de cursos pré-vestibulares reagem a situações estressoras que a preparação para 0 Concurso Vestibular tende a desencadear, como o excesso de estudo aliado à pressão pessoal e social. Buscou-se, assim, evidenciar as manifestações desse estado emocional de estresse.

Constatou-se que a maioria dos estudantes sofria de estresse e, em grande parte, apresentava-0 predominantemente na forma psicológica, não 0 apresentando na forma física. Ressalta-se que tal separação entre físico e psicológico é um conceito usado pelos pesquisadores devido às especificidades do instrumento utilizado nessa pesquisa, que tem como intuito definir o tipo de manifestação do estresse que o indivíduo apresenta. Verificou-se que as somatizaçõesnão foram umaconstanteno presente estudo. Tal constatação mostra a importância de se dar atenção ao lado psicológico do ser, pois o aparelho psíquico também sofre, embora, parte das vezes, as manifestações não se deem de forma tão evidente quanto uma alteração orgânica.

O conceito de saúde da O MS (O rganização Mundial da Saúde) de 1948, está ultrapassado. Para ele, a saúde é uma "situação de perfeito bem-estar físico, mental e social", ou seja, a ausência de qualquer mal-estar, físico ou psicológico. Nota-se a existência de uma relação entre o bem e 0 mal: a saúde é determinada pela doença (Segre, 1997, p. 359).

Partindo da visão da OMS, o homem é um todo integrado, composto de diversas partes (física, mental, social) queinteragem entre si e com o mundo. 0 que se percebe como alternativa válida é tratar as dificuldades através de uma visão interdisciplinar. 0 papel dos profissionais da área da saúde, em especial os psicólogos, éfundamental no sentido de esclarecer e alertar o indivíduo quanto aos possíveis males que um sintoma psicológico pode causar. 0 sintoma é uma manifestação de sofrimento ou atéuma forma de pedir ajuda. Assim, considera-se importante que este receba acompanhamento e dedicação.

0 Concurso Vestibular é um ritual de passagem para os jovens brasileiros, uma barreira a ser transposta, a qual é considerada 0 marco que separa a adolescência da vida adulta. 0 que se pode analisar na sociedade brasileira atual é a grande competitividade existente em relação ao ingresso em uma universidade federal. Há cada vez mais estudantes competindo por um número escasso de vagas. A meritocracia faz com que aqueles que conseguem ingressar no ensino superior sintam-se recompensados, ao contrário dos que não conquistaram uma vaga desejada. Isso cria e fixa uma segregação irreal, entre os "capazes" e os "incapazes" deatingirarealização profissional plena.

0 vestibular está entrelaçado à ideia do projeto de vida pessoal. Nele, o estudante elabora a ideia de aprender uma profissão e se tornar reconhecido. Cria-se a ilusão de que quem é aprovado no concurso tem mais chances de atingir tal realidade. 0 vestibular torna-se um marco único de realização profissional.

Assim,éimportantearealização deestudos relacionados à escolha profissional, ao período de ingresso na faculdade e à entrada na vida adulta, a fim de ampliar o campo de conhecimento dessa área. Sugerir formas de estudo e metodologias alternativas pode auxiliar na diminuição dos danos sociais e psicológicos que esse momento da vida tende a provocar, bem como produzir conhecimentos contextualizados que possam analisar e sugerir ações que favoreçam a aquisição de hábitos adequados de estudo e de autocuidado.

\section{AGRADECIMENTOS}

Agradecemos ao professor Brasílio Ricardo Cirillo da Silva, da Pontifícia Universidade do Rio Grande do Sul, pelos seus ensinamentos e ajuda na parte estatística deste trabalho, ao aluno Guilherme Wendt do grupo de Pós-Graduação: Avaliação e Intervenção no Ciclo Vital, daFaculdadedePsicologia da Pontifícia Universidade do Rio Grande do Sul, pelo auxílio na utilização da ferramenta SPSS e aos dois cursos pré-vestibulares de Porto Alegre que permitiram a coleta de dados em suas sedes. 


\section{REFERÊNCIAS}

Andrade, J. D. (2002). O consumidor e o direito educacional. Revista Eletrônica de Direito Educacional, 1(2), 1-11.

Ballone, G. J., Neto, E. P., \& O tolani, I. V. (2002). Da emoção à lesão: Um guia de medicina psicossomática. São Paulo: Manole

Bardin, L. (1991). Análise de conteúdo. Lisboa: Edições 70.

Cabral, A. P. T., Luna, J. F., Souza, K. N., Macedo, L. M., Mendes, M. G. A., Medeiros, P. A. S., \& Gomes, R. M. (1997). O estresse e as doenças psicossomáticas. Revista de Psicofisiologia, 1(1), 1-22.

Calais, S. L., Andrade, L. M. B., \& Lipp, M. E. N. (2003). Diferenças de sexo e escolaridade na manifestação de stress em adultos jovens. Psicologia: Reflexão e Crítica, 16(2), 257-263.

Castro, M. G., Andrade, T. M. R., \& Muller M. C. (2006). Conceito mente e corpo através da história. Psicologia e estudo, 11(1), 39-43.

Campos, E. P. (1992). 0 paciente somático no grupo terapêutico. In J. M. Filho (O rg.). Psicossomática Hoje. Porto Alegre: Artes Médicas.

Cataldo, N. A., Gauer, G. J. C., \& Furtado, N. R. (O rg.). (2003). Psiquiatria para estudantes de medicina. Porto Alegre: EDIPUCRS.

Hutz, C. S., \& Bardagir, M. P. (2006). Indecisão profissional, ansiedade e depressão na adolescência: a influência dos estilos parentais. PsicoUSF. 11(1), 65-73. Recuperado em 14 março 2007. da SciELO (Scientific Eletronocnic Library on line): w w w. s c i e l o.b v s - p si . o r g. b r / scielo.php?script $=$ sci arttext\&pid $=$ S1413$82712006000100008 \& \operatorname{lng}=p t \& n r m=i s 0$

Last C. G., \& Beidel D. C. (1995). Ansiedade. In M. Lewis (Ed.). Tratado de psiquiatria da infância e adolescência. (pp. 297-308). Porto Alegre: Artes Médicas.

Levenfus, R. S. (1997). Vestibular: ritual de passagem. In R. S. Levenfus, D. H. Soares, I. C. T. Lucchiari, M. D. Silva, M. C. P. Lisboa, \& M. Lassance, et al. (Ed.). Psicodinâmica da escolha profissional. (pp. 198-204). Porto Alegre: Artes Médicas.
Lipp, M. E. N., \& Novaes; L. E . (2000). 0 stress: São Paulo: Contexto.

Margis, R., Picon, P., Cosner, A. N., \& Silveira, R. O. (2003). Relação entre estressores, estresse e ansiedade. Revista de Psiquiatria do Rio Grande do Sul, 25(1), 65-74.

Martins, H. H. H., \& Imbert, M. L. C. (2004). Uma intervenção integradora: A família vai à escola numa $N$ ova Proposta de 0 rientação Profissional. Porto Alegre: Estudos Avançados em O rientação Profissional CAPSO P/ UFRG S.

Papalia, D. E., \& Olds, S. W. (2000). Desenvolvimento humano (7a ed.). Porto Alegre: Artmed. Traduzido por D aniel Bueno.

Rocha, T. H. R., Ribeiro, J. E. C., Pereira, G. A. P., Aveiro, C. C., \& Silva, L. C. A. (2006). Sintomas depressivos em adolescentes de um colégio particular. Psico-USF, 11(11), 95-102.

Santos, L. M. M. (2005). O papel da família e dos pares na escolha profissional. Psicologia em Estudo, 10(1), 57-66.

Segre, M., \& Ferraz, F. C. O. (1997). Conceito de saúde. Revista Saúde Pública, 31(5). 538-542. Recuperado em 30 ago. 2007. da SciELO (Scientific Eletronocnic Library On line): w w w. scielosp.org/ scielo.ph ? script =sci arttext\&pid=S0034$89101997000600016 \& \operatorname{lng}=$ en $\& n r m=i s 0$

Silva, A. L. P., \& Soares, D. H. P. (2001). A orientação profissional como rito preliminar de passagem: sua importância clínica. Psicologia em Estudo, 6(2), 115-121.

Universidade Federal do Rio Grande do Sul (2007). Concurso Vestibular 2007: N úmeros de candidatos por vaga em 1 à 0 pção. Recuperado em 03 jan. 2007. da UFRG S (Universidade Federal do Rio Grande do Sul On line): www.ufrgs.br/ coperse/ cv2007/ densidade2007.htm

Recebido: 18/ 04/ 2008 Received: 04/ 18/ 2008

Aprovado: 02/ 07/ 2008 A pproved: 07/ 02/ 2008 